\title{
TRACHEAL TRANSPLANTATION FOR CARINAL RECONSTRUCTION IN DOGS
}

Katsunobu Kawahara, MD

Kouji Inutsuka, MD

Masafumi Hiratsuka, MD

Satoshi Makihata, MD

Kann Okabayashi, MD,

Takeshi Shiraishi, MD

Takayuki Shirakusa, MD
Background: Experimental carinal allotransplantation has been performed with tracheocarinal Y-shaped allografts in dogs. In this study we tried canine carinal reconstruction with cylindrical allografts. Material and methods: Carinal reconstruction was performed with allotransplantation of cylindrical trachea in dogs, and graft healing was evaluated by bronchoscopic observation, mucosal blood flow measurement, and histologic examination. A section of the recipient carina containing five tracheal rings and two main stem bronchi was removed, and a donor trachea seven rings long was inserted between the recipient trachea and the left main stem bronchus; then side-to-end anastomosis was performed between the graft midportion and recipient right main stem bronchus (new carina). The grafts were wrapped with pedicled omentum. Fresh grafts were transplanted into one group of $\operatorname{dogs}(n=8)$, and grafts cryopreserved for 1 week were transplanted into another group $(n=7)$. Results: No anastomotic leakage occurred in any dog. Excellent healing of grafts and graft anastomoses was observed by fiberoptic bronchoscopy in six dogs $(75 \%)$ in the fresh graft group and in four dogs $(57 \%)$ in the cryopreserved graft group. The mucosal blood flow in the new carina decreased remarkably and, although it recovered, mucosal blood flow remained under the preoperative level on day 28 after the operation. Conclusion: Cylindrical tracheal allotransplantation is useful for carinal reconstruction, and the method of side-to-end anastomosis between the donor trachea and recipient bronchus is a feasible and accessible procedure in dogs. ( $\mathrm{J}$ Thorac Cardiovase Surg 1998;116:397-401)
P reviously we $\mathrm{e}^{1,2}$ documented sufficient viability and good healing in canine immediate tracheocarinal autotransplantation and allotransplantation. In our previous experiments, canine cryopreserved tracheocarinal allografts healed well in $70 \%$ of experimental dogs, and revascularization of grafts derived from the omentum occurred on day $5 .^{2}$ The tracheal membrane blood flow was lower at the midportion of the graft than at the proximal or distal anastomoses, but it had recovered to normal level 4 weeks after the operation. ${ }^{2}$

In this present study we tried carinal reconstruc-

From the Second Department of Surgery, Fukuoka University School of Medicine, Fukuoka, Japan.

Received for publication Jan. 19, 1998; revisions requested March 25, 1998; revisions received April 20, 1998; accepted for publication April 21, 1998.

Address for reprints: Katsunobu Kawahara, MD, Second Department of Surgery, Fukuoka University School of Medicine, Jyohnannku Nanakuma 7-45-1, 814-01, Fukuoka, Japan.

Copyright (c) 1998 by Mosby, Inc.

$0022-5223 / 98 \$ 5.00+0 \quad \mathbf{1 2 / 1 / 9 1 1 7 8}$ tion with allotransplantation of fresh and cryopreserved cylindrical trachea in dogs; the procedure involved inserting a cylindrical graft between the recipient trachea and left main stem bronchus and performing side-to-end anastomosis between the graft midportion and the recipient right main stem bronchus. We evaluated the healing of anastomoses at the midportion of the grafts where mucosal blood flow was lower than it was in the proximal or distal ends.

\section{Materials and methods}

Adult mongrel dogs were used as both donors and recipients. All dogs were maintained according to the National Society at Medical Research Principles of Laboratory Animal Care. This study was approved by our institutional animal care and review committee.

Donor dogs. Adult mongrel dogs weighing from 10 to $17 \mathrm{~kg}$ were anesthetized and intubated. A right thoracotomy was performed, and the trachea and the carina were isolated. A seven-ring length of mediastinal trachea was harvested.

Cryopreservation. The tracheas were put in sterilized bottles and stored in a freezer at $-80^{\circ} \mathrm{C}$ for 1 to 2 weeks. 

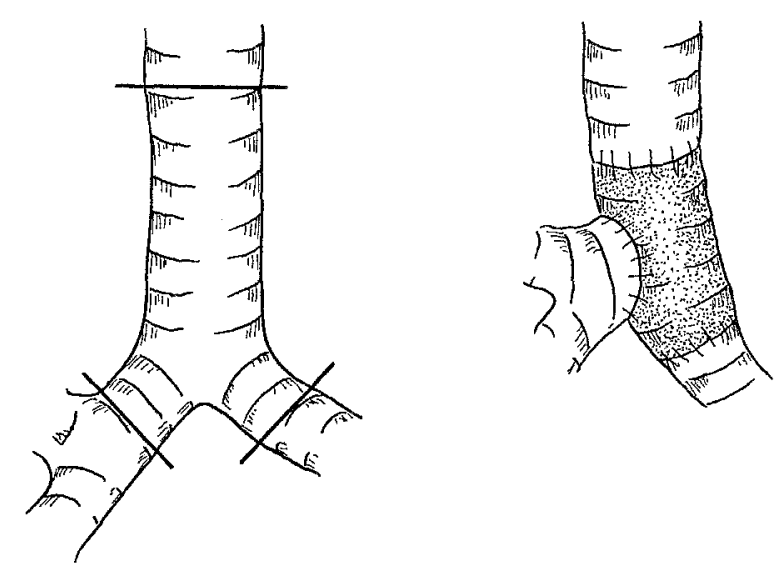

Fig. 1. Surgical procedure and a site of mucosal blood flow measurement.

Before transplantation they were rewarmed at $35^{\circ} \mathrm{C}$ for 15 minutes.

Recipient dogs. Recipient dogs of the same weight as the donor dogs were put in a left decubitus position. An upper median laparotomy was performed, and a pedicled omental flap was made and pulled into the right pleural cavity through the diaphragm. Then a right thoracotomy was performed. The azygos vein was transected, and the trachea and carina were isolated. The right main bronchus two rings distal to the carina was transected and intubated through the pleural cavity. Subsequently, the right lung was ventilated, and five rings of the trachea and two rings of the left main bronchus were transected; then the trachea and carina were removed en bloc.

The dogs were classified into two groups. In group 1 $(n=8)$, initially a cylindrical graft of a donor trachea without cryopreservation was inserted between the recipient trachea and the left main stem bronchus. Subsequently, an orotracheal tube was inserted into the left main bronchus, and the left lung was ventilated. An oval window was opened at the cartilaginous wall in the midportion of the graft, and the right main bronchus was anastomosed to the window (Fig. 1). After the anastomosis was completed, the graft was wrapped with the pedicled omentum through the diaphragmatic route. In group $2(n=7)$, a cylindrical graft cryopreserved for 1 week was inserted by means of the same procedure described for the group 1 dogs.

Recipient dogs were given antibiotics for 1 week after the operation. Immunosuppressive therapy with administration of FK506, $1.0 \mathrm{mg} / \mathrm{kg}$, was performed for 4 weeks after the operation and then discontinued.

Bronchoscopic examinations were performed and mucosal blood flows of the grafts at the anastomoses of the proximal site, distal site, and midportion were measured by the hydrogen gas clearance technique ${ }^{3}$ after the operation. The anastomotic healing of grafts was examined weekly.

When the recipient dogs were killed, the grafis werc removed and examined histologically with hematoxylin and eosin staining. The anastomotic healing of the graft

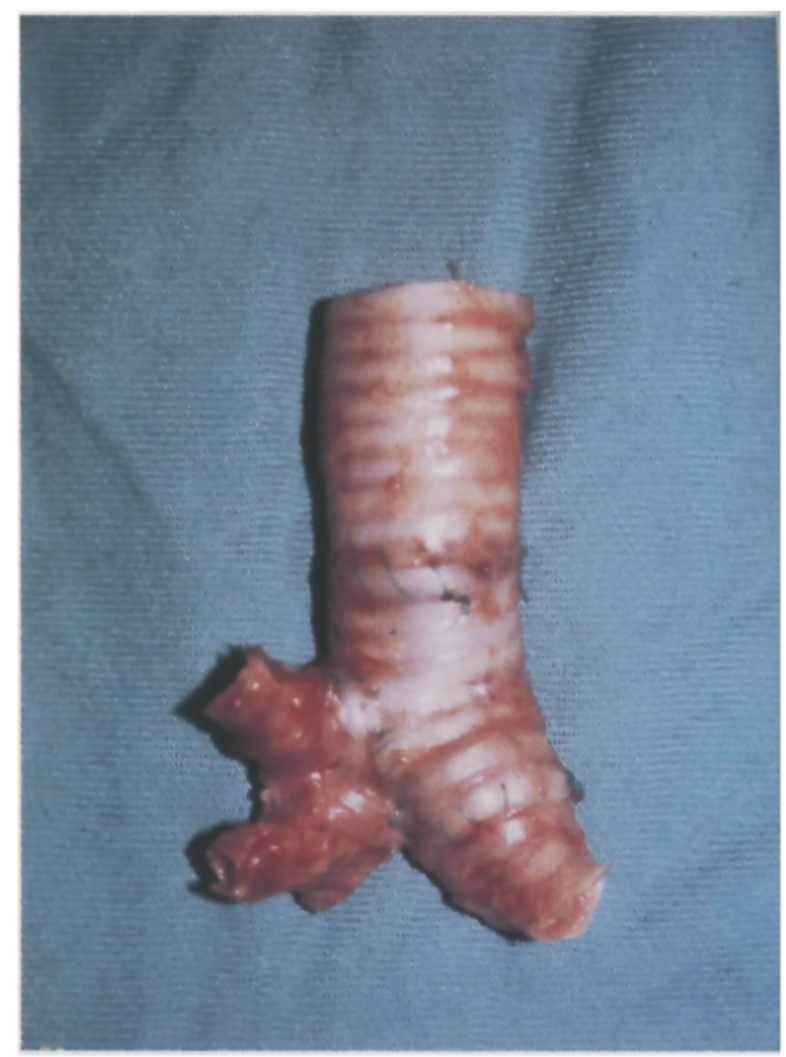

Fig. 2. Macroscopic photograph of the graft on postoperative day 40 . The omentum with which the graft had been wrapped was removed.

midportion was histologically evaluated by the wound healing score system of Nakanishi and associates. ${ }^{4}$

\section{Results}

Within 15 days after the operation, one dog in group 1 and two dogs in group 2 died as a result of the FK506 administration. Two dogs in group 1 and one dog in group 2 died of pneumonia or respiratory failure with airway stenosis resulting from graft failure. In group 1, three dogs survived for 46 to 74 days, and two dogs are alive 286 and 686 days after the operation (Table I). Survival in group 1 was five of eight dogs $(62 \%)$, with $70 \%$ confidence limits from $38 \%$ to $83 \%$. In group 2 , two dogs survived for 34 and 56 days after the operation, and two dogs were alive for 313 and 553 days after the operation (Table 1). Survival in group 2 was four of seven dogs $(57 \%)$, with $70 \%$ confidence linits from $32 \%$ to $82 \%$.

No anastomotic lcakage occurred in any dog. Excellent healing of graft and graft anastomoses was observed by fiberoptic bronchoscopy in six of eight 


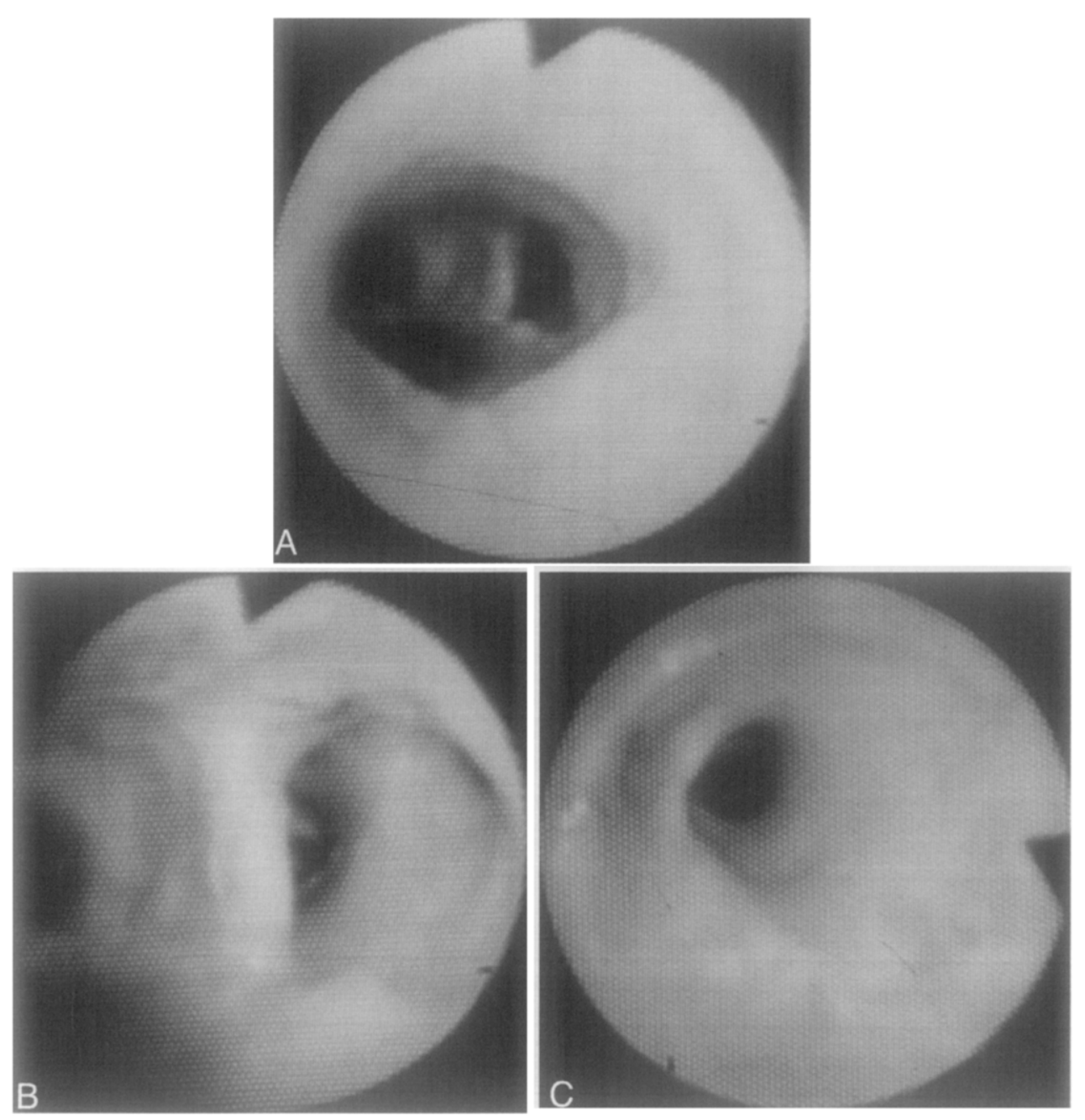

Fig. 3. Bronchofiberscopic findings of the anastomoses in the graft: Proximal anastomosis (A), new carina (B), and distal anastomosis (C) are shown.

Table I. Carinal reconstruction with canine tracheal allotransplantation

\begin{tabular}{|c|c|c|c|c|c|}
\hline Subject & $\begin{array}{l}\text { Survival time } \\
\text { (days) }\end{array}$ & $\begin{array}{l}\text { Fiberoptic } \\
\text { graft status }\end{array}$ & $\begin{array}{c}\text { Histologic } \\
\text { healing score }\end{array}$ & $\begin{array}{c}\text { Duration of } \\
\text { immunosuppression }\end{array}$ & Cause of death \\
\hline \multicolumn{6}{|c|}{ Immediatc transplantation } \\
\hline 1. & 7 & Edema & 15 & Until death & Pneumonia \\
\hline 2. & 17 & Malacia & 16 & Until death & Respiratory failure \\
\hline 3. & 21 & Healed & 8 & Until death & Wasting \\
\hline 4. & 46 & Healed & 6 & 30 days & Killed \\
\hline 5. & 66 & Healed & 7 & 28 days & Killed \\
\hline 6. & 74 & Healed & 7 & 32 days & Killed \\
\hline 7. & 286 & Healed & - & 28 days & Alive \\
\hline 8. & 686 & Healed & - & 37 days & Alive \\
\hline \multicolumn{6}{|c|}{ Cryopreserved transplantation } \\
\hline 1. & 8 & Edema & 15 & Until death & Wasting \\
\hline 2. & 16 & Edema & 12 & Until death & Wasting \\
\hline 3. & 22 & Malacia & 16 & Until death & Respiratory failure \\
\hline 4. & 34 & Healed & 9 & 28 days & Killed \\
\hline 5. & 56 & Healed & 5 & 30 days & Killed \\
\hline 6. & 313 & Healed & - & 33 days & Alive \\
\hline 7. & 553 & Healed & - & 28 days & Alive \\
\hline
\end{tabular}




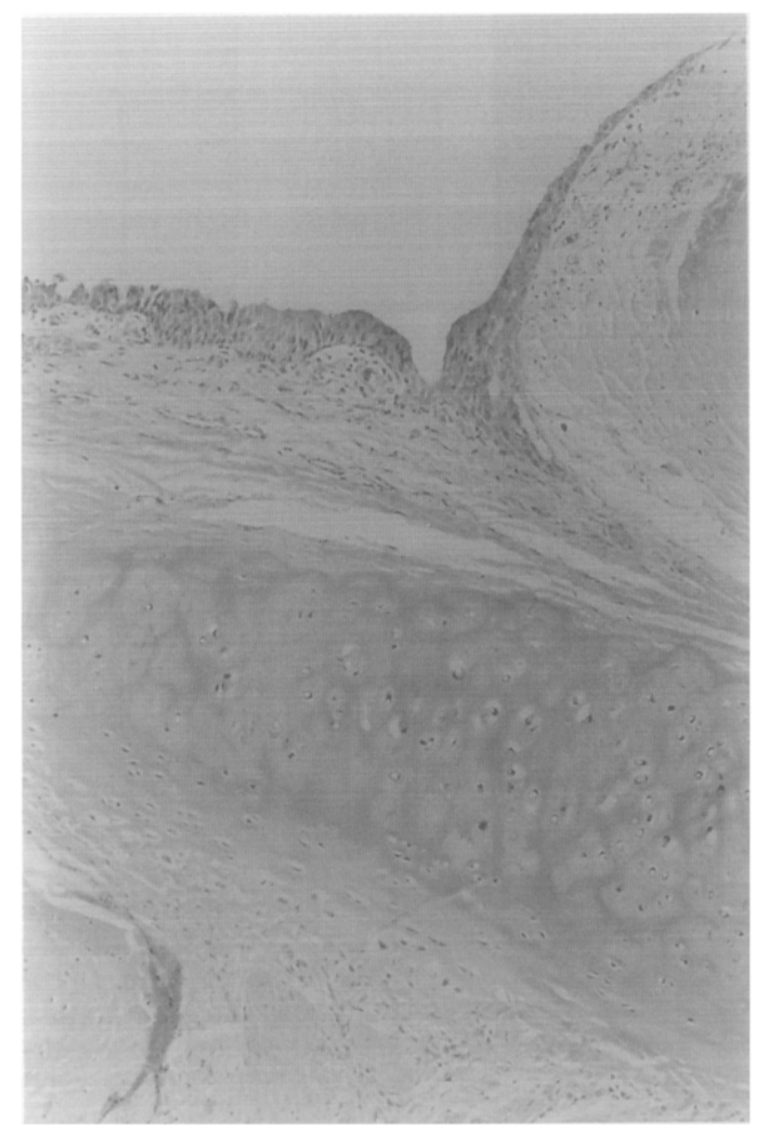

Fig. 4. Microscopic photograph of the anastomosis at the new carina. Mononuclear cell infiltration and a granulation tissue-like reaction with fibrosis are absent. Normal mucociliary epithelium covers the graft, and no damage to the tracheal glands or cartilage is obscrved.

dogs $(75 \%)$ in group 1 , with $70 \%$ confidence limits from $50 \%$ to $90 \%$, and in four of seven dogs $(57 \%)$ in group 2 , with $70 \%$ confidence limits from $32 \%$ to $80 \%$ (Figs. 2 and 3 ).

The histologic healing score of the dogs, except living dogs, was 6,6 , and 7 in group 1 and 5 and 9 in group 2 (Table I). Microscopic findings are shown in Fig. 4. Epithelialization of the anastomosis was accomplished within approximately 3 weeks of transplantation in all groups. The healing score in the dogs with graft failure was 15 and 16 in group 1 and 12,15 , and 16 in group 2.

Histologically, cartilaginous degeneration and necrosis of the grafts were observed in the dogs that died of graft failure. The mononuclear cell infiltration was limited.

The mucosal blood flow at the graft anastomoses decreased from day 3 to day 7 after the operation,
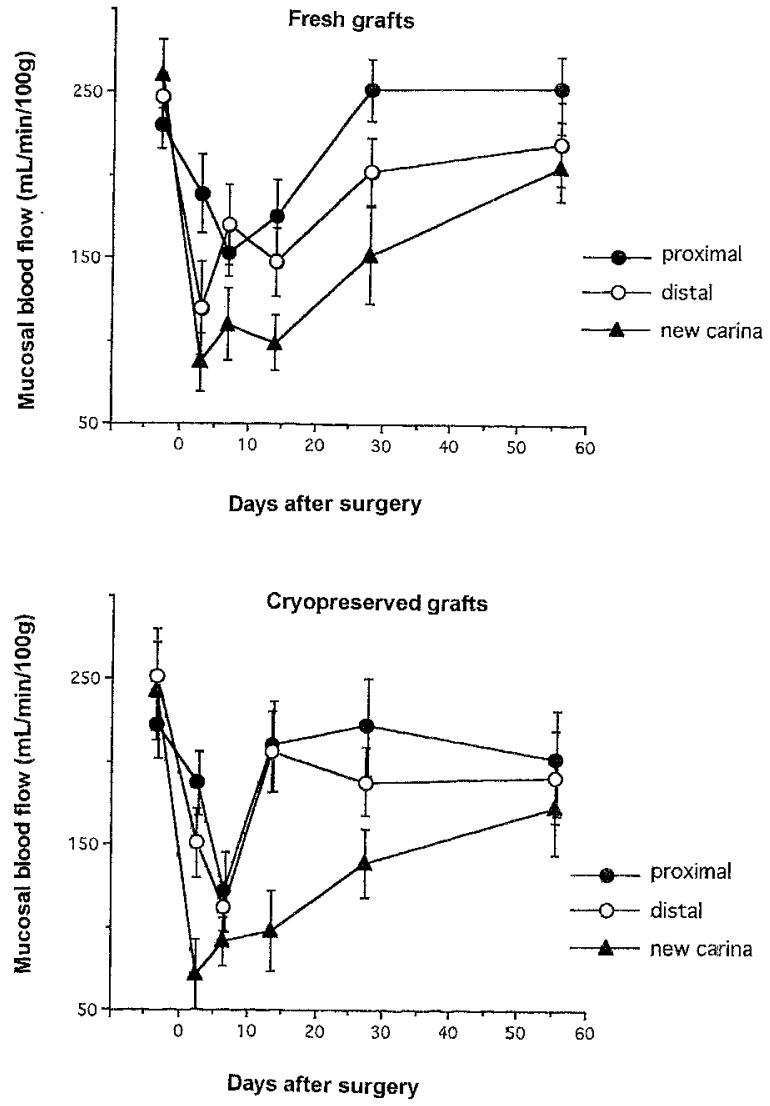

Fig. 5. Mucosal blood flow of fresh grafts (top) and cryopreserved grafts (bottom).

and then it recovered to preoperative levels after day 28 in both the groups. The mucosal blood flow of the grafts at the anastomosis between the graft and the right main stem bronchus (new carina) decreased remarkably after transplantation and then increased gradually, but it remained under the preoperative level on day 28.

\section{Discussion}

In this study, we demonstrated that a cylindrical allograft of the trachea was useful for carinal reconstruction involving allotransplantation of the canine trachea. Although mucosal blood flow in the midportion of a cylindrical graft was lower than that in the proximal or distal site of the graft, a side-to-end anastomosis between the midportion of the allograft and the recipient bronchus was feasible, and graft healing was excellent. We estimated the dehiscence in the side-to-end anastomosis of the graft and recipient bronchus because, in our previous study, the tracheal mucosal blood flow was lower at the 
midportion of the grafts than that at the proximal or distal anastomotic site of the grafts; however, dehiscence did not occur in this current study. Yokomise and colleagues ${ }^{5}$ reported that neovascularization of a $10 \mathrm{~cm}$ long graft was promoted by omentum insertion at the midportion of the graft. Kitada ${ }^{6}$ reported that a circular incision in the long graft further enhanced the blood supply to the midportion of the graft. In our study, although the omentum was not inserted, revascularization originating from the omentum could be accomplished rapidly at the side-to-end anastomosis at the midportion of the graft (new carina); therefore, anastomotic dehiscence did not occur. It normally takes 2 to 3 weeks after lung transplantation for bronchial circulation to be restored by means of vascular growth across the healing bronchial anastomosis. Collateral circulation between omental vessels and bronchial vessels distal to the bronchial anastomosis had been accomplished by 4 days after the operation. ${ }^{7}$ Nakanishi, Shirakusa, and Takachi ${ }^{8}$ reported that omentopexy is an effective method to facilitate neovascularization in tracheal allografts. Therefore, to restore tracheal circulation and protect the anastomosis, we instituted the procedure of wrapping a pedicle of omentum around the tracheal anastomosis after its completion. Blood flow of the omentum and vascularization of the tracheal adventitia are very important factors for success of tracheal allotransplantation. The omentum should not be twisted or stretched when it is brought into the chest and wrapped around the graft. Furthermore, connective tissues surrounding the trachea should not be dissected when the grafts are harvested.

Cylindrical grafts are convenient because they can be used for any reconstruction of the central airway. A Y-shaped graft can be used only for carinal reconstruction. Furthermore, more than two cylindrical allografts can be harvested from one donor, but only one Y-shaped allograft can be harvested from one donor.

In canine tracheal allotransplantation, grafts resisted rejection and immunosuppression when only a short course of mizoribine was administered after transplantation, allowing the possibility of long-term viability. ${ }^{9}$ Yokomise and colleagues ${ }^{10}$ reported that immunosuppressant-free canine tracheal allotransplantation was consistently possible after long-term cryopreservation of a graft in a preservative solution containing trehalose. In our study, an immunosuppressant was administered for 4 weeks after the operation and then discontinued. After discontinuation of immunosuppressant, graft failure was not observed in either dogs with fresh grafts or those with grafts cryopreserved for 1 week.

We conclude that allotransplantation of a cylindrical trachea is useful for carinal reconstruction and that the side-to-end anastomosis between donor trachea and recipient bronchus is a feasible and reliable procedure in dogs.

\section{REFERENCES}

1. Takachi T, Shirakusa T, Shiraishi T, Okabayashi K, Inutsuka K, Kawahara K, et al. Experimental carinal autotransplantation and allotransplantation. J Thorac Cardiovasc Surg 1995; $110: 762-7$.

2. Inutsuka K, Kawahara K, Takachi T, Okabayashi K, Shiraishi $T$, Shirakusa T. Reconstruction of trachea and carina with immediate or cryopreserved allografts in dogs. Ann Thorac Surg 1996;62:1480-4.

3. Aukland K, Bower BF, Berliner RW. Measurement of local blood flow with hydrogen gas. Circ Res 1964;14:164-87.

4. Nakanishi R, Shirakusa T, Hanagiri T. Early histopathologic features of tracheal allotransplant rejection: a study in nonimmunosuppressed dogs. Transplant Proc 1994;26:3715-8.

5. Yokomise H, Inui K, Wada H, Ueda $M$, Hitomi S, Ito $H$. Split transplantation of the trachea: a new operative procedure for extended tracheal resection. $J$ Thorac Cardiovasc Surg 1996;112:314-8.

6. Kitada M. Study of revascularization of tracheal graft in a canine autotransplantation model-a novel technique to promote blood supply to a long graft. Nippon Kyoubu Geka Gakkai Zasshi 1996;44:2011-8.

7. Morgan E, Lima O, Goldberg M, Ferdman A, Luk SK, Cooper JD. Successful revascularization of totally ischemic bronchial autografts with omental pedicle flaps in dogs. J Thorac Cardiovase Surg 1982;84:204-10.

8. Nakanishi R, Shirakusa T, Takachi T. Omentopexy for tracheal autografts. Ann Thorac Surg 1994;57:841-5.

9. Nakanishi R, Yasumoto K, Shirakusa T. Short-course immunosuppression after tracheal allotransplantation in dogs. J Thorac Cardiovasc Surg 1995;109:910-17.

10. Yokomise $\mathrm{H}$, Inui $\mathrm{K}$, Wada $\mathrm{H}$, Ueda $\mathrm{M}$, Hitomi S. Long-term cryopreservation can prevent rejection of canine tracheal allografts with preservation of graft viability. I Thorac Cardiovasc Surg 1996;111:930-4. 